\title{
Synthesis of Some Bioactive Sulfonamide and Amide Derivatives of Piperazine Incorporating Imidazo[1,2-B]Pyridazine Moiety
}

\author{
Ashish Bhatt*, Ravi Kant and Rajesh K Singh
}

Department of Chemistry, Mewar University, Chittorgarh, Rajasthan, India

\begin{abstract}
Some new sulfonamide and amide derivatives containing piperazine ring and imidazo[1,2-b]pyridazine moiety have been synthesized by the reaction of 6-chloro-2-substituted aryl(or alkyl)imidazo[1,2-b]pyridazine derivatives [obtained by the reaction of 3-amino-6-chloro pyridazine with 2-bromo-1-substituted aryl(or alkyl)ethanone] with homopiperazine in NMP and followed by reaction with alkyl (or substituted aryl) acid chloride or sulfonyl chloride in presence of triethyl amine and dichloromethane. All the synthesized compounds were characterized by elemental analysis, ${ }^{1} \mathrm{H}$ NMR and LCMS. These were screened for in vitro antimicrobial activity against two gram positive (Bacillus subtilis and Staphylococcus aureus) and two gram negative bacteria (Pseudomonas fluorescens and Escherichia coli), as well as for antifungal and antimalarial activity.
\end{abstract}

Keywords: Imidazo[1,2-b]pyridazine; Homopiperazine; Antimicrobial and antimalarial activity

\section{Introduction}

Sulfonamide and amide derivatives comprise an important class of drugs with diverse biological applications. Sulfonamides are widely used as antimicrobial [1,2], anticancer [3,4], anti-inflammatory [5] and antiviral agents as well as HIV protease inhibitors [6]. Sulfonamides were the first effective chemotherapeutic agents to be utilized efficiently to prevent and cure the bacterial infection in human beings [7-10].

The piperazine moiety appears in many drugs encompassing a broad range of activities (e.g., Oxatomide, Almitrine, Hydroxyzine, Buclizine, Lomerizine [11]. Nitrogen in piperazine ring plays an important role in exerting biological effects. The basicity of piperazine nitrogen plays an important role in selectivity and potency towards the biological targets. This moiety (monoaryl and diarylpiperazine) also found in drug candidates displaying anti-allergenic [12], antibacterial [13], anti-anxiety [14], anti-emetic [15], antimigraine [16] and platelet antiaggregatory activities [17]. In addition, piperazine moiety is present in many cardiovascular drugs [18] (e.g., Manidipine, Doxazosin, Trimetazidine, Flunarizine, Prazosin) and drug candidates [19,20]. Piperazine and their derivatives also possess antimalarial activity [21], antioxidative activity [22] and antifungal activity [23] and found in many drug molecules such as Meclizine (motion sickness drug), Cyclizine (antiemetic and antihistamine), Clozapine (antipsychotic drug), Imatinib (leukemia drug), Befuraline (stimulant and antidepressant), Antrafenine (analgesic), Trazodone (sedating antidepressant) and Niaprazine (sedating antihistamine) etc.

Piperazine and sulfonamide derivatives represent a category of pharmacologically interesting compounds having diverse biological activities. Intensive research has been carried out on the synthesis and analysis of pharmacological activities of these derivatives. Substituted sulfonamide derivatives are important category of pharmacophores that have a wide spectrum of pharmaceutical accomplishments: as antimalarial [24], anti-microbial [25], anti-bacterial [26,27], anti-cancer [28] anti-fungal [29], anti-oxidant [30], anti-HIV [31], antiplasmodial [32], anti-neoplastic [33], anti-proliferative [34] activities and additionally known to act as 5-HT6, 5-HT7 receptor antagonists [35,36], A2B and CXCR3 antagonists [37,38],11 $\beta$-HSD [39], histone deacetylase (HDAC) inhibitors [40], $\beta$-secretase (BACE1) inhibitors [41] and dual PI3K/mTOR inhibitors [42].

The chemistry of pyridazines and their fused heterocyclic derivatives has received considerable attention owing to their synthetic and effective biological importance. Pyridazines have been reported to possess antimicrobial [43-45], antituberculosis [46-48], antifungal [49], anticancer [50], anti-hypertensive [51], herbicidal [52], antiinflammatory [53] activities and protein tyrosine phosphatise 1B(PTP1B) inhibitors [54]. They also have an immense potential in agricultural science as plant growth regulators and crop protection agents [55]. The incorporation of two moieties increases biological activity of both and thus it was of value to synthesize some new heterocyclic derivatives having two moieties in the same molecules.

Several imidazo [1,2-b] pyridazine derivative have demonstrated biological activity including inhibitors of the central nervous system [56], antipyritic and hypothermal activity [57], anticonvulsant activity, analgesic and antispasmotic activity [58-60].

Looking at the importance of these heterocyclic nuclei, it is thought of interest to accommodate sulphonamide and amide of piperazine with imidazo[1,2-b] pyridazine moieties in single molecular framework and screen them for their various biological activities.

\section{Materials and Methods}

\section{General procedures}

Reagent grade chemicals were used without further purification. All the melting points were taken in open capillaries and are uncorrected. The purity and mass of the synthesized compounds was checked by LCMS. $1 \mathrm{H}$ NMR spectral was recorded in $\mathrm{CDCl}_{3} / \mathrm{DMSO}$ with tetramethylsilane (TMS) as the internal standard at $400 \mathrm{MHz}$ on a Bruker DRTX-400 spectrophotometer. The chemical shifts are reported as parts per million (ppm). Elemental analysis was performed using a (EURO EA 3000 instrument). Acme silica gel-G and Merck silica gel (100 to 200, 60 to 120 meshes) were used for analytical TLC and Column chromatography respectively

*Corresponding author: Ashish Bhatt, Department of Chemistry, Mewar University, Chittorgarh, Rajasthan-312 901, India, Tel: +917742845501; E-mail: itsbhatt2007@yahoo.co.in

Received April 11, 2016; Accepted April 19, 2016; Published April 24, 2016

Citation: Bhatt A, Kant R, Singh RK (2016) Synthesis of Some Bioactive Sulfonamide and Amide Derivatives of Piperazine Incorporating Imidazo[1,2-B] Pyridazine Moiety. Med chem (Los Angeles) 6: 257-263. doi:10.4172/21610444.1000355

Copyright: ( 2016 Bhatt A, et al. This is an open-access article distributed under the terms of the Creative Commons Attribution License, which permits unrestricted use, distribution, and reproduction in any medium, provided the original author and source are credited. 


\section{Chemistry}

We have prepared some novel sulfonamide or amide derivatives of piperazine ring incorporating imidazo[1,2-b]pyridazine moiety in three steps, using 3-amino-6-chloro pyridazine, 2-bromo-1substituted aryl(or alkyl)ethanone, homopiperazine and alkyl (or substituted aryl) acid chloride or sulfonyl chloride as the starting materials. 3-amino-6-chloro pyridazine were treated with 2-bromo1-substituted aryl(or alkyl)ethanone in ethanol to obtain 6-chloro-2substituted aryl(or alkyl)imidazo[1,2-b]pyridazine $1(\mathrm{a}-\mathrm{d})$ which on reaction with homopiperazine in NMP results 6-(piperazin-1-yl)-2substituted aryl(or alkyl)imidazo[1,2-b]pyridazine 2(a-d) and further by reacting with alkyl (or substituted aryl) acid chloride or sulfonyl chloride in presence of triethyl amine in Dichloromethane results the desired sulfonamide or amide derivatives. The clear procedure for the preparation of desired sulfonamide or amide derivatives are given below.

Preparation of novel sulfonamide and amide derivatives of piperazine incorporating imidazo $[1,2-b]$ pyridazine moiety

General procedure for the synthesis of 6-chloro-2-substituted aryl(or alkyl)imidazo[1,2-b]pyridazine: To a solution of 3-amino-6chloro pyridazine $(0.01 \mathrm{~mole})$ in ethanol $(10 \mathrm{~mL})$ was added 2-bromo1 -substituted aryl (or alkyl) ethanone at room temperature. Then the reaction mixture was refluxed at $80^{\circ} \mathrm{C}$ for $4 \mathrm{hrs}$. The reaction mixture was then cooled and poured into ice-cold water. The resulting precipitate was filtered, washed several times with water, dried and recrystallized from ethanol.

\section{Spectral data of intermediate}

6-chloro-2-(trifluoromethyl)imidazo[1,2-b]pyridazine $\quad 1(a)$ : ${ }^{1} \mathrm{H}-\mathrm{NMR}\left(400 \mathrm{MHz}, \mathrm{CDCl}_{3}\right.$ ): 9.05 (s, 1H, Imidazo-H), 8.36 (d, J=9.6 $\mathrm{Hz}, 1 \mathrm{H}$, pyridazine- $\mathrm{H}), 7.59$ (d, J=9.6 Hz, $1 \mathrm{H}$, pyridazine- $\mathrm{H})$. MS: 222.3 $\left(\mathrm{M}^{+}\right)$. Anal. Calcd for $\mathrm{C}_{7} \mathrm{H}_{3} \mathrm{ClF}_{3} \mathrm{~N}_{3}: \mathrm{C}-37.95 \%, \mathrm{H}-1.36 \%$, Cl- $16.00 \%$, F-25.72\%, N-18.96. Found: C- 37.85\%, H- 1.33\%, Cl-15.96\%, F-25.70\%, N-18.92.

6-chloro-2-p-tolylimidazo[1,2-b]pyridazine $\quad$ 1(b): $\quad{ }^{1} \mathrm{H}-\mathrm{NMR}$ $\left(400 \mathrm{MHz}, \mathrm{CDCl}_{3}\right): 8.85$ (s, $1 \mathrm{H}$, Imidazo-H), $8.20(\mathrm{~d}, \mathrm{~J}=9.6 \mathrm{~Hz}, 1 \mathrm{H}$, pyridazine-H), 7.93 (d, J=8.4 Hz, 2H, Ar-H), 7.35 (d, J=9.6 Hz, $1 \mathrm{H}$, pyridazine-H), 7.28 (d, J=8.0 Hz,2H, Ar-H), 2.34 (s,3H, Ar- $\left.\mathrm{CH}_{3}\right)$. MS: $244.4\left(\mathrm{M}^{+}\right)$. Anal. Calcd for $\mathrm{C}_{13} \mathrm{H}_{10} \mathrm{ClN}_{3}$ : C- 64.07\%, H- $4.14 \%, \mathrm{Cl}-$ 14.55\%, N-17.24. Found: C- $64.04 \%, \mathrm{H}-4.11 \%, \mathrm{Cl}-14.52 \%, \mathrm{~N}-17.21 \%$.

6-chloro-2-(4-(trifluoromethyl)phenyl)imidazo[ $1,2-b]$ pyridazine 1(c): ${ }^{1} \mathrm{H}-\mathrm{NMR}\left(400 \mathrm{MHz}, \mathrm{CDCl}_{3}\right)$ : 9.05 (s, $1 \mathrm{H}$, Imidazo-H), 8.26-8.23 (m, 3H), $7.83(\mathrm{~d}, \mathrm{~J}=8.0 \mathrm{~Hz}, 2 \mathrm{H}, \mathrm{Ar}-\mathrm{H}), 7.41(\mathrm{~d}, \mathrm{~J}=9.6 \mathrm{~Hz}$, $1 \mathrm{H}$, pyridazine-H). MS: $298.2\left(\mathrm{M}^{+}\right)$. Anal. Calcd for $\mathrm{C}_{13} \mathrm{H}_{7} \mathrm{ClF}_{3} \mathrm{~N}_{3}$ : C52.45\%, H- 2.37\%, Cl-11.91\%, F-19.15\%, N-14.12\%. Found: C- $52.42 \%$, H- $2.33 \%$, Cl-11.89\%, F-19.14\%, N-14.09\%.

6-chloro-2-(2,5-dichlorophenyl)imidazo[1,2-b]pyridazine $\mathbf{1 ( d )}$ ${ }^{1} \mathrm{H}-\mathrm{NMR}\left(400 \mathrm{MHz}, \mathrm{CDCl}_{3}\right): 8.92$ (s, $1 \mathrm{H}$, Imidazo-H), 8.30 (d, J=9.2 $\mathrm{Hz}, 1 \mathrm{H}$, pyridazine-H), 8.21-8.20 (m, $1 \mathrm{H}, \mathrm{Ar}-\mathrm{H}), 7.64(\mathrm{~d}, \mathrm{~J}=8.8 \mathrm{~Hz}, 1 \mathrm{H}$, Ar-H), 7.51-7.46 (m, 2H). MS: $298.4\left(\mathrm{M}^{+}\right)$. Anal. Calcd for $\mathrm{C}_{12} \mathrm{H}_{6} \mathrm{Cl}_{3} \mathrm{~N}_{3}$ : C- $48.28 \%$, H- 2.03\%, Cl-35.62\%, N-14.07\%. Found: C- $48.25 \%$, H$1.99 \%, \mathrm{Cl}-35.60 \%, \mathrm{~N}-14.03 \%$.

General procedure for the synthesis of 6-(piperazin-1-yl)-2substituted aryl(or alkyl)imidazo[1,2-b]pyridazine

The mixture of 6-chloro-2-substituted aryl(or alkyl)imidazo[1,2-b] pyridazine ( 0.01 mole) and homopiperazine $(0.05$ mole) in NMP (5 $\mathrm{mL}$ ) was heated at $150^{\circ} \mathrm{C}$ for $1 \mathrm{hr}$. The reaction mixture was diluted with water and extracted with ethyl acetate. The organic layer was washed with water, brine and dried over $\mathrm{Na}_{2} \mathrm{SO}_{4}$. The solvent was evaporated and crude compound was used as such for next step without any purification.

\section{Spectral data of intermediate}

6-(piperazin-1-yl)-2-(trifluoromethyl)imidazo[1,2-b] pyridazine 2(a): ${ }^{1} \mathrm{H}-\mathrm{NMR}\left(400 \mathrm{MHz}, \mathrm{CDCl}_{3}\right): 8.50(\mathrm{~s}, 1 \mathrm{H}$, Imidazo-H), $7.91(\mathrm{~d}, \mathrm{~J}=10.0 \mathrm{~Hz}, 2 \mathrm{H}$, pyridazine- $\mathrm{H}), 7.37 \quad(\mathrm{~d}, \mathrm{~J}=10.0 \mathrm{~Hz}, 2 \mathrm{H}$, pyridazine-H), 3.43-3.41 (m, $4 \mathrm{H}$, piperazine- $\mathrm{H}), 3.34$ (bs, $1 \mathrm{H}, \mathrm{NH})$, 2.80-2.78(m, $4 \mathrm{H}$, piperazine-H), MS:272.6 $\left(\mathrm{M}^{+}\right)$. Anal. Calcd for $\mathrm{C}_{11} \mathrm{H}_{12} \mathrm{~F}_{3} \mathrm{~N}_{5}$ : C- $48.71 \%, \mathrm{H}-4.46 \%, \mathrm{~F}-21.01 \%, \mathrm{~N}-25.82 \%$. Found: C$48.67 \%$, H- $4.42 \%$, F-20.98\%, N-25.79\%.

6-(piperazin-1-yl)-2-p-tolylimidazo[1,2-b]pyridazine 2(b): ${ }^{1} \mathrm{H}-\mathrm{NMR}\left(400 \mathrm{MHz}, \mathrm{CDCl}_{3}\right): 8.40(\mathrm{~s}, 1 \mathrm{H}$, Imidazo-H), 7.83-7.80 (m,3H), $7.21(\mathrm{~d}, \mathrm{~J}=8.0 \mathrm{~Hz}, 2 \mathrm{H}, \mathrm{Ar}-\mathrm{H}), 7.15(\mathrm{~d}, \mathrm{~J}=10.0 \mathrm{~Hz}, 1 \mathrm{H}$, pyridazine- $\mathrm{H})$, 3.39-3.37 (m, $4 \mathrm{H}$, piperazine-H), 3.34 (bs, $1 \mathrm{H}, \mathrm{NH}), 2.82-2.79(\mathrm{~m}, 4 \mathrm{H}$, piperazine-H), $2.31(\mathrm{~s}, 3 \mathrm{H}, \mathrm{Ar}-\mathrm{H})$. MS: $294.4\left(\mathrm{M}^{+}\right)$. Anal. Calcd for $\mathrm{C}_{17} \mathrm{H}_{19} \mathrm{~N}_{5}$ : C- $69.60 \%, \mathrm{H}-6.53 \%, \mathrm{~N}-23.87 \%$. Found: C- $69.58 \%, \mathrm{H}-$ $6.51 \%, \mathrm{~N}-23.83 \%$.

6-(piperazin-1-yl)-2-(4-(trifluoromethyl)phenyl)imidazo[1,2-b] pyridazine $2(\mathrm{c}):{ }^{1} \mathrm{H}-\mathrm{NMR}\left(400 \mathrm{MHz}, \mathrm{CDCl}_{3}\right): 8.02(\mathrm{~s}, 1 \mathrm{H}$, Imidazo-H), $7.97(\mathrm{~d}, \mathrm{~J}=8.4 \mathrm{~Hz}, 2 \mathrm{H}, \mathrm{Ar}-\mathrm{H}), 7.80(\mathrm{~d}, \mathrm{~J}=10.0 \mathrm{~Hz}, 1 \mathrm{H}$, pyridazine- $\mathrm{H})$, $7.62(\mathrm{~d}, \mathrm{~J}=8.4 \mathrm{~Hz}, 2 \mathrm{H}, \mathrm{Ar}-\mathrm{H}), 7.30(\mathrm{~d}, \mathrm{~J}=10.0 \mathrm{~Hz}, 1 \mathrm{H}$, pyridazine- $\mathrm{H})$, 3.40-3.37 (m, $4 \mathrm{H}$, piperazine-H), 3.34 (bs, $1 \mathrm{H}, \mathrm{NH}), 2.81-2.78(\mathrm{~m}, 4 \mathrm{H}$, piperazine-H). MS: $348.1\left(\mathrm{M}^{+}\right)$. Anal. Calcd for $\mathrm{C}_{17} \mathrm{H}_{16} \mathrm{~F}_{3} \mathrm{~N}_{5}: \mathrm{C}-58.78 \%$, H- 4.64\%, F-16.41\%, N-20.16\%. Found: C- 58.76\%, H- 4.61\%, F-16.37\%, $\mathrm{N}-20.13 \%$.

2-(2,5-dichlorophenyl)-6-(piperazin-1-yl)imidazo[1,2-b] pyridazine 2(d): ${ }^{1} \mathrm{H}-\mathrm{NMR}\left(400 \mathrm{MHz}, \mathrm{CDCl}_{3}\right): 8.46(\mathrm{~s}, 1 \mathrm{H}$, Imidazo-H), $8.20(\mathrm{~d}, \mathrm{~J}=2.4 \mathrm{~Hz}, 1 \mathrm{H}, \mathrm{Ar}-\mathrm{H}), 7.89(\mathrm{~d}, \mathrm{~J}=10.0 \mathrm{~Hz}, 1 \mathrm{H}$, pyridazine- $\mathrm{H}), 7.57$ (d, J=8.8 Hz,1H, Ar-H), 7.39 (dd, J1=8.6 Hz, J2=2.6Hz,1H, Ar-H), 7.27 (d, J=10.0 Hz, $1 \mathrm{H}$, pyridazine- $\mathrm{H}), 3.43-3.40(\mathrm{~m}, 4 \mathrm{H}$, piperazine- $\mathrm{H}), 3.33$ (bs, $1 \mathrm{H}, \mathrm{NH}), 2.82-2.79\left(\mathrm{~m}, 4 \mathrm{H}\right.$, piperazine-H). MS: $348.5\left(\mathrm{M}^{+}\right)$. Anal. Calcd for $\mathrm{C}_{16} \mathrm{H}_{15} \mathrm{Cl}_{2} \mathrm{~N}_{5}$ : C- 55.19\%, H- 4.34\%, Cl-20.36\%, N-20.11\%. Found: C- $55.16 \%$, H- $4.31 \%, \mathrm{Cl}-20.33 \%, \mathrm{~N}-20.09 \%$.

General procedure for the conversion of 6-(piperazin-1-yl)-2substituted aryl(or alkyl)imidazo[1,2-b]pyridazine into alkyl (or substituted aryl) sulfonamide or amide 3(a-j)

To a solution of 6-(piperazin-1-yl)-2-substituted aryl(or alkyl) imidazo[1,2-b]pyridazine (0.01 mole) in Dichloromethane was added triethyl amine ( 0.015 mole) followed by alkyl (or substituted aryl) acid chloride or sulfonyl chloride $(0.013$ mole $)$ at $0^{\circ} \mathrm{C}$ and stirred the reaction mixture at room temperature for $2 \mathrm{hrs}$. The reaction mixture was diluted with water and extracted with Dichloromethne. The organic layer was washed with water, brine and dried over $\mathrm{Na}_{2} \mathrm{SO}_{4}$. The solvent was evaporated and crude compound was purified by using column chromatography with 100-200 silica gel to give compound 3(aj) (Scheme 1).

\section{Spectral data of Desired sulfonamide and amide derivatives}

1-(4-(2-(trifluoromethyl)imidazo[1,2-b]pyridazin-6-yl) piperazin-1-yl)propan-1-one 3(a): ${ }^{1} \mathrm{H}-\mathrm{NMR}\left(400 \mathrm{MHz}, \mathrm{CDCl}_{3}\right)$ : $8.54(\mathrm{~s}, 1 \mathrm{H}$, Imidazo-H), 7.97 (d, J=10.0Hz,1H, pyridazine- $\mathrm{H}), 7.41(\mathrm{~d}$, $\mathrm{J}=10.0 \mathrm{~Hz}, 1 \mathrm{H}$, pyridazine- $\mathrm{H}), 3.58-3.51(\mathrm{~m}, 8 \mathrm{H}$, piperazine- $\mathrm{H}), 2.37$ (q, J=7.4 Hz, 2H, $\left.-\mathrm{COCH}_{2}\right), 1.00\left(\mathrm{t}, \mathrm{J}=7.4 \mathrm{~Hz}, 3 \mathrm{H},-\mathrm{CH}_{3}\right)$. LCMS: 328.4 $\left(\mathrm{M}^{+}\right)$, Purity-97.5\%. Anal. Calcd for $\mathrm{C}_{14} \mathrm{H}_{16} \mathrm{~F}_{3} \mathrm{~N}_{5} \mathrm{O}$ : C- $51.37 \%, \mathrm{H}-$ 4.93\%, F-17.41\%, N-21.40\%, O-4.89\%. Found: C- 51.33\%, H- 4.91\%, F-17.39\%, N-21.37\%, O-4.87\%. 


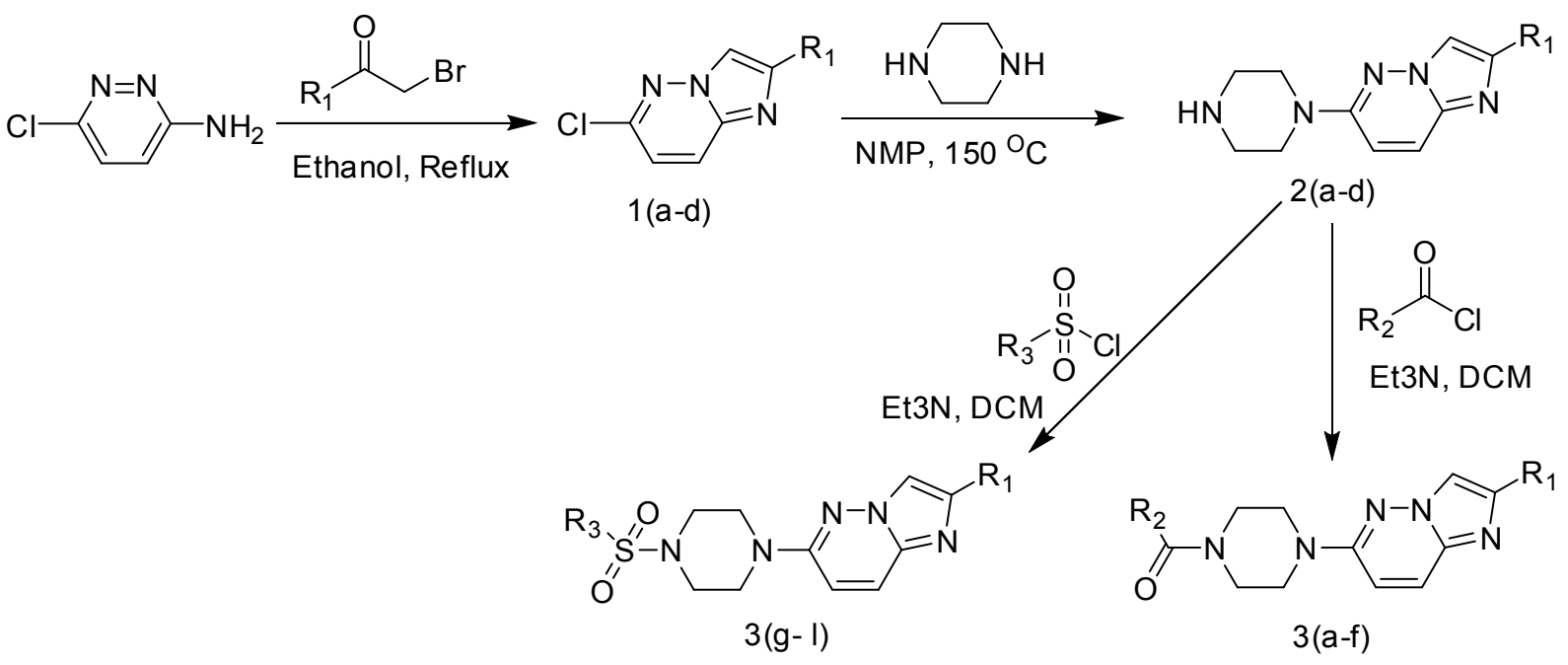

$\mathrm{R}_{1}=\mathrm{CF}_{3}, 4 \mathrm{CH}_{3}$ - phenyl, 4- $\mathrm{CF}_{3}$ phenyl, 2,4-Dichloro phenyl

$\mathrm{R}_{2}=$ Ethyl, p- phenyl, 4-CF 3 phenyl

p-tolyl(4-(2-(trifluoromethyl)imidazo[1,2-b]pyridazin-6-yl) piperazin-1-yl)methanone 3(b): ${ }^{1} \mathrm{H}-\mathrm{NMR}\left(400 \mathrm{MHz}, \mathrm{CDCl}_{3}\right): 7.93$ (s,1H, Imidazo-H), $7.78(\mathrm{~d}, \mathrm{~J}=10.0 \mathrm{~Hz}, 1 \mathrm{H}$, pyridazine-H), 7.35 (d, J=8.0 $\mathrm{Hz}, 2 \mathrm{H}, \mathrm{Ar}-\mathrm{H}), 7.24$ (d, J=8.0 Hz,2H, Ar-H), 6.95(d, J=10.0 Hz,1H, pyridazine- $\mathrm{H}), 3.88-3.56(\mathrm{~m}, 8 \mathrm{H}$, piperazine- $\mathrm{H}), 2.40\left(\mathrm{~s}, 3 \mathrm{H}, \mathrm{Ar}-\mathrm{CH}_{3}\right)$. LCMS: $390.2\left(\mathrm{M}^{+}\right)$, Purity-98.2\%. Anal. Calcd for $\mathrm{C}_{19} \mathrm{H}_{18} \mathrm{~F}_{3} \mathrm{~N}_{5} \mathrm{O}$ : C58.61\%, H- 4.66\%, F-14.64\%, N-17.99\%, O-4.11\%, Found: C- 58.58\%, H- $4.63 \%$, F-14.61\%, N-17.96\%, O-4.09\%.

(4-(2-(trifluoromethyl)imidazo[1,2-b]pyridazin-6-yl)piperazin1-yl)(4-(trifluoromethyl) phenyl) methanone (3c): ${ }^{1} \mathrm{H}-\mathrm{NMR}$ (400 MHz, $\left.\mathrm{CDCl}_{3}\right): 7.94(\mathrm{~s}, 1 \mathrm{H}$, Imidazo-H), $7.81(\mathrm{~d}, \mathrm{~J}=10.0 \mathrm{~Hz}, 1 \mathrm{H}$, pyridazine-H), $7.72(\mathrm{~d}, \mathrm{~J}=8.0 \mathrm{~Hz}, 2 \mathrm{H}, \mathrm{Ar}-\mathrm{H}), 7.57(\mathrm{~d}, \mathrm{~J}=8.0 \mathrm{~Hz}, 2 \mathrm{H}$, Ar-H), $6.94(\mathrm{~d}, \mathrm{~J}=10.0 \mathrm{~Hz}, 1 \mathrm{H}$, pyridazine-H), 3.95-3.56 (m, $8 \mathrm{H}$, piperazine-H). LCMS: $444.3\left(\mathrm{M}^{+}\right)$, Purity-95.7\%. Anal. Calcd for $\mathrm{C}_{19} \mathrm{H}_{15} \mathrm{~F}_{6} \mathrm{~N}_{5} \mathrm{O}: \mathrm{C}-51.47 \%, \mathrm{H}-3.41 \%, \mathrm{~F}-25.71 \%, \mathrm{~N}-15.80 \%, \mathrm{O}-3.61 \%$, Found: C- $51.44 \%, \mathrm{H}-3.39 \%$, F-25.68\%, N-15.77\%, O-3.57\%.

1-(4-(2-p-tolylimidazo[1,2-b]pyridazin-6-yl)piperazin-1yl)propan-1-one (3d): ${ }^{1} \mathrm{H}-\mathrm{NMR}\left(400 \mathrm{MHz}, \mathrm{CDCl}_{3}\right): 7.97(\mathrm{~s}, 1 \mathrm{H}$, Imidazo-H), 7.80 (d, J=8.0 Hz,2H, Ar-H), $7.75(\mathrm{~d}, \mathrm{~J}=10.0 \mathrm{~Hz}, 1 \mathrm{H}$, pyridazine-H), $7.24(\mathrm{~d}, \mathrm{~J}=8.4 \mathrm{~Hz}, 2 \mathrm{H}, \mathrm{Ar}-\mathrm{H}), 6.79(\mathrm{~d}, \mathrm{~J}=9.6 \mathrm{~Hz}, 1 \mathrm{H}$, pyridazine-H), 3.80-3.71 (m,2H, piperazine- $\mathrm{H}), 3.64-3.62(\mathrm{~m}, 2 \mathrm{H}$, piperazine- $\mathrm{H}), \quad 3.55-3.53(\mathrm{~m}, 2 \mathrm{H}$, piperazine- $\mathrm{H}), 3.49-3.47(\mathrm{~m}, 2 \mathrm{H}$, piperazine-H), $2.41\left(\mathrm{q}, \mathrm{J}=7.4 \mathrm{~Hz}, 2 \mathrm{H},-\mathrm{COCH}_{2}\right), 2.38\left(\mathrm{~s}, 3 \mathrm{H}, \mathrm{Ar}-\mathrm{CH}_{3}\right)$, 1.19 (t, J=7.4 Hz, 3H,-CH $)$. LCMS: $350.3\left(\mathrm{M}^{+}\right)$, Purity-96.4\%. Anal. Calcd for $\mathrm{C}_{20} \mathrm{H}_{23} \mathrm{~N}_{5} \mathrm{O}$ : C- $68.47 \%, \mathrm{H}-6.63 \%, \mathrm{~N}-20.04 \%$, O-4.58\%, Found: C-68.45\%, H- 6.60\%, N-20.01\%, O-4.54\%.

1-(4-(2-(4-(trifluoromethyl)phenyl)imidazo[1,2-b]pyridazin-6yl)piperazin-1-yl)propan-1-one (3e): ${ }^{1} \mathrm{H}-\mathrm{NMR}\left(400 \mathrm{MHz}, \mathrm{CDCl}_{3}\right)$ : 8.05 (s,1H, Imidazo-H), 8.01 (d, J=8.4 Hz,2H, Ar-H), 7.77 (d, J=10.0 $\mathrm{Hz}, 1 \mathrm{H}$, pyridazine- $\mathrm{H}), 7.67$ (d, J=8.4 Hz, 2H, Ar-H), 6.85 (d, J=9.6 $\mathrm{Hz}, 1 \mathrm{H}$, pyridazine- $\mathrm{H}), \quad 3.81-3.78(\mathrm{~m}, 2 \mathrm{H}$, piperazine- $\mathrm{H}), \quad 3.64-3.63$ $(\mathrm{m}, 2 \mathrm{H}$, piperazine- $\mathrm{H}), \quad 3.57-3.55(\mathrm{~m}, 2 \mathrm{H}$, piperazine- $\mathrm{H}), \quad 3.52-3.49$ (m, $2 \mathrm{H}$, piperazine-H), $2.41\left(\mathrm{q}, \mathrm{J}=7.4 \mathrm{~Hz}, 2 \mathrm{H},-\mathrm{COCH}_{2}\right), 1.19$ (t, J=7.4 $\left.\mathrm{Hz}, 3 \mathrm{H},-\mathrm{CH}_{3}\right)$. LCMS: $404.2\left(\mathrm{M}^{+}\right)$, Purity-98.4\%, Anal. Calcd for $\mathrm{C}_{20} \mathrm{H}_{20} \mathrm{~F}_{3} \mathrm{~N}_{5} \mathrm{O}: \mathrm{C}-59.55 \%, \mathrm{H}-5.00 \%, \mathrm{~F}-14.13 \%, \mathrm{~N}-17.36 \%$, O-3.97\%, Found: C-59.53\%, H- 4.97\%, F-14.11\%, N-17.33\%, O-3.94\%. 1-(4-(2-(2,5-dichlorophenyl)imidazo[1,2-b]pyridazin-6-yl) piperazin-1-yl)propan-1-one (3f): ${ }^{1} \mathrm{H}-\mathrm{NMR}\left(400 \mathrm{MHz}, \mathrm{CDCl}_{3}\right): 8.44$ (s,1H, Imidazo-H), 8.26 (d, J=2.4 Hz,1H, Ar-H), 7.77 (d, J=10.0 Hz,1H, pyridazine-H), 7.38 (d, J=8.4 Hz, $1 \mathrm{H}, A r-H), 7.20(\mathrm{dd}, \mathrm{J} 1=8.4 \mathrm{~Hz}$, $\mathrm{J} 2=2.4 \mathrm{~Hz}, 1 \mathrm{H}, \mathrm{Ar}-\mathrm{H}), 6.87(\mathrm{~d}, \mathrm{~J}=10.0 \mathrm{~Hz}, 1 \mathrm{H}$, pyridazine- $\mathrm{H}), 3.81-3.78$ $(\mathrm{m}, 2 \mathrm{H}$, piperazine- $\mathrm{H}), \quad 3.64-3.62(\mathrm{~m}, 2 \mathrm{H}$, piperazine- $\mathrm{H}), \quad 3.58-3.56$ $(\mathrm{m}, 2 \mathrm{H}$, piperazine- $\mathrm{H}), 3.52-3.49(\mathrm{~m}, 2 \mathrm{H}$, piperazine- $\mathrm{H}), 2.41(\mathrm{q}, \mathrm{J}=7.4$ $\left.\mathrm{Hz}, 2 \mathrm{H},-\mathrm{COCH}_{2}\right), 1.19\left(\mathrm{t}, \mathrm{J}=7.4 \mathrm{~Hz}, 3 \mathrm{H},-\mathrm{CH}_{3}\right)$. LCMS: $404.3\left(\mathrm{M}^{+}\right)$, Purity-99.07\%, Anal. Calcd for $\mathrm{C}_{19} \mathrm{H}_{19} \mathrm{Cl}_{2} \mathrm{~N}_{5} \mathrm{O}: \mathrm{C}-56.45 \%, \mathrm{H}-4.74 \%$, Cl-17.54\%, N-17.32\%, O-3.96\%, Found: C-56.42\%, H- $4.71 \%$, Cl$17.52 \%, \mathrm{~N}-17.29 \%$, O-3.93\%

6-(4-(ethylsulfonyl)piperazin-1-yl)-2-(trifluoromethyl) imidazo[1,2-b]pyridazine (3g): ${ }^{1} \mathrm{H}-\mathrm{NMR}\left(400 \mathrm{MHz}, \mathrm{CDCl}_{3}\right): 7.95$ $(\mathrm{s}, 1 \mathrm{H}$, Imidazo-H), $7.79(\mathrm{~d}, \mathrm{~J}=10.0 \mathrm{~Hz}, 1 \mathrm{H}$, pyridazine- $\mathrm{H}), 6.93(\mathrm{~d}$, $\mathrm{J}=10.0 \mathrm{~Hz}, 1 \mathrm{H}$, pyridazine- $\mathrm{H}), 3.65-3.62(\mathrm{~m}, 4 \mathrm{H}$, piperazine- $\mathrm{H}), 3.46-$ $3.43(\mathrm{~m}, 4 \mathrm{H}$, piperazine- $\mathrm{H}), 3.00\left(\mathrm{q}, \mathrm{J}=7.4 \mathrm{~Hz}, 2 \mathrm{H},-\mathrm{SO}_{2} \mathrm{CH}_{2}\right), 1.40(\mathrm{t}$, $\left.\mathrm{J}=7.4 \mathrm{~Hz}, 3 \mathrm{H},-\mathrm{CH}_{3}\right)$. LCMS: $364.2\left(\mathrm{M}^{+}\right)$, Purity-97.39\%. Anal. Calcd for $\mathrm{C}_{13} \mathrm{H}_{16} \mathrm{~F}_{3} \mathrm{~N}_{5} \mathrm{O}_{2} \mathrm{~S}: \mathrm{C}-42.97 \%$, H- 4.44\%, F-15.69\%, N-19.27\%, O-8.81\%, S-8.82\%. Found: C-42.93\%, H- 4.41\%, F-15.66\%, N-19.25\%, O- $8.79 \%$, $\mathrm{S}-8.78 \%$

6-(4-tosylpiperazin-1-yl)-2-(trifluoromethyl)imidazo[1,2-b] pyridazine (3h): ${ }^{1} \mathrm{H}-\mathrm{NMR}\left(400 \mathrm{MHz}, \mathrm{CDCl}_{3}\right): 7.90(\mathrm{~s}, 1 \mathrm{H}$, Imidazo-H), $7.73(\mathrm{~d}, \mathrm{~J}=10.0 \mathrm{~Hz}, 1 \mathrm{H}$, pyridazine-H), 7.66 (d, J=8.0 Hz, 2H, Ar-H), $7.34(\mathrm{~d}, \mathrm{~J}=8.0 \mathrm{~Hz}, 2 \mathrm{H}$, Ar- $\mathrm{H}), 6.84(\mathrm{~d}, \mathrm{~J}=10.0 \mathrm{~Hz}, 1 \mathrm{H}$, pyridazine- $\mathrm{H})$, 3.64-3.61 (m, $4 \mathrm{H}$, piperazine- $\mathrm{H}), 3.15-3.12(\mathrm{~m}, 4 \mathrm{H}$, piperazine- $\mathrm{H}), 2.42$ (s,3H, Ar- $\left.\mathrm{CH}_{3}\right)$. LCMS: $426.2\left(\mathrm{M}^{+}\right)$, Purity-99.73\%. Anal. Calcd for $\mathrm{C}_{18} \mathrm{H}_{18} \mathrm{~F}_{3} \mathrm{~N}_{5} \mathrm{O}_{2} \mathrm{~S}$ : C- $50.82 \%$, H- 4.26\%, F-13.40\%, N-16.46\%, O-7.52\%, S-7.54\%, Found: C- 50.79\%, H- 4.23\%, F-13.37\%, N-16.43\%, O-7.49\%, S-7.51\%.

2-(trifluoromethyl)-6-(4-(4-(trifluoromethyl)phenylsulfonyl) piperazin-1-yl)imidazo[1,2-b] pyridazine (3i): ${ }^{1} \mathrm{H}-\mathrm{NMR}(400 \mathrm{MHz}$, $\left.\mathrm{CDCl}_{3}\right)$ : 7.93-7.91 (m,3H), 7.83 (d, J=8.0 Hz, 2H, Ar-H), 7.75 (d, J=10.0 $\mathrm{Hz}, 1 \mathrm{H}$, pyridazine- $\mathrm{H}), 6.84$ (d, J=10.0 Hz,1H, pyridazine- $\mathrm{H})$, 3.66$3.64(\mathrm{~m}, 4 \mathrm{H}$, piperazine- $\mathrm{H}), 3.21-3.19(\mathrm{~m}, 4 \mathrm{H}$, piperazine- $\mathrm{H})$. LCMS: $480.3\left(\mathrm{M}^{+}\right)$, Purity-94.54\%. Anal. Calcd for $\mathrm{C}_{18} \mathrm{H}_{15} \mathrm{~F}_{6} \mathrm{~N}_{5} \mathrm{O}$ S: C- $45.10 \%$, H- 3.15\%, F-23.78\%, N-14.61\%, O-6.67\%, S-6.69\%. Found: C- 45.09\%, H- 3.13\%, F-23.75\%, N-14.59\%, O-6.65\%, S-6.66\%. 
6-(4-(ethylsulfonyl)piperazin-1-yl)-2-p-tolylimidazo[1,2-b] pyridazine (3j): ${ }^{1} \mathrm{H}-\mathrm{NMR}\left(400 \mathrm{MHz}_{\mathrm{CDCl}}\right): 7.97(\mathrm{~s}, 1 \mathrm{H}$, Imidazo-H), $7.80(\mathrm{~d}, \mathrm{~J}=8.0 \mathrm{~Hz}, 2 \mathrm{H}, \mathrm{Ar}-\mathrm{H}), 7.75$ (d, J=10.0 Hz,1H, pyridazine-H), $7.24(\mathrm{~d}, \mathrm{~J}=8.4 \mathrm{~Hz}, 2 \mathrm{H}, \mathrm{Ar}-\mathrm{H}), 6.78(\mathrm{~d}, \mathrm{~J}=10.0 \mathrm{~Hz}, 1 \mathrm{H}$, pyridazine- $\mathrm{H})$, 3.62-3.60 (m,4H, piperazine- $\mathrm{H}), 3.46-3.43(\mathrm{~m}, 4 \mathrm{H}$, piperazine- $\mathrm{H}), 3.00$ (q, J=7.4 Hz, 2H,-SO $\left.\mathrm{CH}_{2}\right), 2.38\left(\mathrm{~s}, 3 \mathrm{H}, \mathrm{Ar}-\mathrm{CH}_{3}\right), 1.40(\mathrm{t}, \mathrm{J}=7.4 \mathrm{~Hz}, 3 \mathrm{H}$,$\mathrm{CH}_{3}$ ). LCMS: $386.3\left(\mathrm{M}^{+}\right)$, Purity-96.27\%. Anal. Calcd for $\mathrm{C}_{19} \mathrm{H}_{23} \mathrm{~N}_{5} \mathrm{O}_{2} \mathrm{~S}$ : C- $59.20 \%$, H- $6.01 \%$, N-18.17\%, O-8.30\%, S-8.32\%, Found: C-59.18\%, H- $6.00 \%, \mathrm{~N}-18.14 \%$, O-8.28\%, S-8.29\%.

6-(4-(ethylsulfonyl)piperazin-1-yl)-2-(4-(trifluoromethyl) phenyl)imidazo[1,2-b]pyridazine (3k):

${ }^{1} \mathrm{H}-\mathrm{NMR}\left(400 \mathrm{MHz}, \mathrm{CDCl}_{3}\right): 8.06(\mathrm{~s}, 1 \mathrm{H}$, Imidazo-H), $8.00(\mathrm{~d}$, $\mathrm{J}=8.0 \mathrm{~Hz}, 2 \mathrm{H}, \operatorname{Ar}-\mathrm{H}), 7.78(\mathrm{~d}, \mathrm{~J}=10.0 \mathrm{~Hz}, 1 \mathrm{H}$, pyridazine- $\mathrm{H}), 7.67(\mathrm{~d}$, $\mathrm{J}=8.0 \mathrm{~Hz}, 2 \mathrm{H}, \mathrm{Ar}-\mathrm{H}), 6.84(\mathrm{~d}, \mathrm{~J}=10.0 \mathrm{~Hz}, 1 \mathrm{H}$, pyridazine- $\mathrm{H}), 3.63-3.62$ $(\mathrm{m}, 4 \mathrm{H}$, piperazine- $\mathrm{H}), 3.47-3.45(\mathrm{~m}, 4 \mathrm{H}$, piperazine- $\mathrm{H}), 3.00(\mathrm{q}, \mathrm{J}=7.2$ $\left.\mathrm{Hz}, 2 \mathrm{H},-\mathrm{SO}_{2} \mathrm{CH}_{2}\right), 1.41\left(\mathrm{t}, \mathrm{J}=7.4 \mathrm{~Hz}, 3 \mathrm{H},-\mathrm{CH}_{3}\right)$. LCMS: $440.3\left(\mathrm{M}^{+}\right)$, Purity-92.91\% Anal. Calcd for $\mathrm{C}_{19} \mathrm{H}_{20} \mathrm{~F}_{3} \mathrm{~N}_{5} \mathrm{O}_{2} \mathrm{~S}$ : C- $51.93 \%$, H- 4.59\%, F-12.97\%, N-15.94\%, O-7.28\%, S-7.30\%, Found: C-51.91\%, H- 4.55\%, F-12.94\%, N-15.91\%, O-7.25\%, S-7.28\%.

2-(2,5-dichlorophenyl)-6-(4-(ethylsulfonyl)piperazin-1-yl) imidazo[1,2-b]pyridazine (31):

${ }^{1} \mathrm{H}-\mathrm{NMR}\left(400 \mathrm{MHz}, \mathrm{CDCl}_{3}\right): 8.44(\mathrm{~s}, 1 \mathrm{H}$, Imidazo-H), $8.26(\mathrm{~d}$, $\mathrm{J}=2.80 \mathrm{~Hz}, 1 \mathrm{H}, \mathrm{Ar}-\mathrm{H}), 7.78(\mathrm{~d}, \mathrm{~J}=10.0 \mathrm{~Hz}, 1 \mathrm{H}$, pyridazine- $\mathrm{H}), 7.38(\mathrm{~d}$, $\mathrm{J}=8.8 \mathrm{~Hz}, 1 \mathrm{H}, \mathrm{Ar}-\mathrm{H}), 7.20$ (dd, J1=8.8 Hz, J2=2.4 Hz,1H, Ar-H), 6.85 $(\mathrm{d}, \mathrm{J}=10.0 \mathrm{~Hz}, 1 \mathrm{H}$, pyridazine- $\mathrm{H}), 3.65-3.62(\mathrm{~m}, 4 \mathrm{H}$, piperazine- $\mathrm{H}), 3.46-$ $3.44(\mathrm{~m}, 4 \mathrm{H}$, piperazine- $\mathrm{H}), 3.00\left(\mathrm{q}, \mathrm{J}=7.4 \mathrm{~Hz}, 2 \mathrm{H},-\mathrm{SO}_{2} \mathrm{CH}_{2}\right), 1.40$ (t, $\left.\mathrm{J}=7.4 \mathrm{~Hz}, 3 \mathrm{H},-\mathrm{CH}_{3}\right)$. LCMS: $440.5\left(\mathrm{M}^{+}\right)$, Purity-98.9\%, Anal. Calcd for $\mathrm{C}_{18} \mathrm{H}_{19} \mathrm{Cl}_{2} \mathrm{~N}_{5} \mathrm{O}_{2} \mathrm{~S}: \mathrm{C}-49.10 \%, \mathrm{H}-4.35 \%, \mathrm{Cl}-16.10 \%, \mathrm{~N}-15.90 \%, \mathrm{O}-7.27 \%$, S-7.28\%, Found: C-49.08\%, H- 4.32\%, Cl-16.07\%, N-15.88\%, O-7.24\%, S-7.25\%.

\section{Antimicrobial activity}

All the synthesized compounds were tested against two gram positive bacteria (Staphylococcus aureus, Streptococcus pyogenes) and two gram negative bacteria (Escherichia coli, Pseudomonas aeruginosa) using micro broth dilution method [61-63] for the determination of minimal inhibition concentration. For the antifungal activity the common standard strains that were used, are C. albicans, $A$. niger and $A$. clavatus. Muller Hinton broth (Microcare laboratory and Tuberculosis Research Centre, Surat-3, India) was used as nutrient medium to grow and dilute the drug suspension for the test bacteria. Inoculum Size for Test Strain was adjust to $10^{8} \mathrm{Cfu}$ [Colony Forming Unit] per milliliter by comparing the turbidity. DMSO was used as diluents / vehicle to get desired concentration of drugs to test upon Standard bacterial strains. Serial dilutions were prepared in primary and secondary screening. In primary screening $1000 \mathrm{micro} / \mathrm{ml}, 500 \mathrm{micro} / \mathrm{ml}$, and $250 \mathrm{micro} /$ $\mathrm{ml}$ concentrations of the synthesized compounds were taken. The active synthesized compounds found in this primary screening were further tested in a second set of dilution against all microorganisms. The highest dilution showing at least $99 \%$ inhibition zone is taken as MIC. The test mixture should contain $10^{8}$ organism $/ \mathrm{ml}$. Standard drugs Ampicillin and Chloramphenicol were used as antibacterial for comparison. Standard drugs Nystatin and Greseofulvin were used as antifungal for comparison.

\section{Antimalarial activity}

The in vitro antimalarial assay was carried out in 96 well microtitre plates according to the microassay protocol reference. The cultures of P. falciparum strain were maintained in medium RPMI 1640 supplemented with $25 \mathrm{mM}$ HEPES, $1 \%$ D-glucose, $0.23 \%$ sodium bicarbonate and $10 \%$ heat inactivated human serum. The asynchronous parasites of $P$. falciparum were synchronized after 5\% D-sorbitol treatment to obtain only the ring stage parasitized cells. For carrying out the assay, an initial ring stage parasitaemia of 0.8 to $1.5 \%$ at $3 \%$ haematocrit in a total volume of $200 \mu$ of medium RPMI-1640 was determined by Jaswant Singh Bhattacharya (JSB) staining to assess the percent parasitaemia (rings) and uniformally maintained with $50 \%$ RBCs $\left(\mathrm{O}^{+}\right)$. A stock solution of $5 \mathrm{mg} / \mathrm{ml}$ of each of the test samples was prepared in DMSO and subsequent dilutions were prepared with culture medium. The diluted samples in $20 \mu \mathrm{l}$ volume were added to the test wells so as to obtain final concentrations (at five fold dilutions) ranging between $0.4 \mu \mathrm{g} / \mathrm{ml}$ to $100 \mu \mathrm{g} / \mathrm{ml}$ in duplicate well containing parasitized cell preparation. The culture plates were incubated at $37^{\circ} \mathrm{C}$ in a candle jar. After 36 to $40 \mathrm{~h}$ incubation, thin blood smears from each well were prepared and stained with JSB stain. The slides were microscopically observed to record maturation of ring stage parasites into trophozoites and schizonts in presence of different concentrations of the test agents. The test concentration which inhibited the complete maturation into schizonts was recorded as the minimum inhibitory concentrations (MIC). Quinine was taken as the reference drug.

\section{Results and Discussion}

\section{Chemistry}

3-amino-6-chloro pyridazine on reaction with 2-bromo-1substituted aryl(or alkyl)ethanone in ethanol gives 6-chloro-2substituted aryl(or alkyl)imidazo[1,2-b]pyridazine 1(a-d). The obtained compound (1) on reaction with homopiperazine in NMP results 6-(piperazin-1-yl)-2-substituted aryl(or alkyl)imidazo[1,2-b] pyridazine $2(\mathrm{a}-\mathrm{d})$ which on further reaction with alkyl (or substituted aryl) acid chloride or sulfonyl chloride gives sulfonamide or amide derivatives of piperazine ring incorporating imidazo[1,2-b]pyridazine moiety $3(\mathrm{a}-1)$. The list of synthesized compound are represented by Table 1.

\section{Antibacterial activity}

The antibacterial activity of all the synthesized compounds were tested in-vitro against pathogenic E. coli, $P$. aeruginosa, S. aureus and $S$. pyogenus and the results were compared with standard drugs (Ampicillin and Chloramphenicol). In case of S. aureus, Compound $3 \mathrm{~b}$ shows higher activity and compounds $3 \mathrm{a}, 3 \mathrm{c}, 3 \mathrm{e}, 3 \mathrm{f}, 3 \mathrm{j}$, and $3 \mathrm{l}$ exhibit good activity while $3 \mathrm{~d}, 3 \mathrm{~g}, 3 \mathrm{~h}, 3 \mathrm{i}$ and $3 \mathrm{k}$ show moderate activity. In case of $S$. pyogenus compounds $3 \mathrm{c}, 3 \mathrm{~d}$ and $3 \mathrm{f}$ exhibit good activity and compound $3 \mathrm{~b}$ show moderate activity while $3 \mathrm{a}, 3 \mathrm{~b}, 3 \mathrm{e}, 3 \mathrm{~g}, 3 \mathrm{~h}, 3 \mathrm{i}, 3 \mathrm{j}, 3 \mathrm{k}$ and 31 possess less activity. In case of E. coli Compound 3d exhibit higher activity and compounds $3 \mathrm{f}, 3 \mathrm{j}$ show moderate activity while rest of the compounds possess less activity. In case of $P$. aeruginosa compounds $3 \mathrm{e}, 3 \mathrm{k}$ and $3 \mathrm{l}$ exhibit good activity than the rest of the compounds. The results are given in Table 2.

\section{Antifungal activity}

The antifungal activity of all the synthesized compounds were tested in-vitro against fungi $C$. albicans, A. niger and A. clavatus and the results were compared with standard drugs (Nystatin and Greseofulvin). In case of C. albicans compound 3a exhibit higher activity and 3c, 3e, 3h, $3 \mathrm{i}$, and $3 \mathrm{l}$, show good activity while compounds rest of the compouns possess less activity. In case of $A$. niger and $A$. clavatus all the compounds possess less activity. The results are given in Table 3.

\section{Antimalarial activity}

For antimalarial activity, Compounds $3 \mathrm{a}, 3 \mathrm{c}$ and $3 \mathrm{k}$ exhibit good activity closer to reference compound Quinine while rest of the compounds possess less activity. The results are given in Table 4. 


\begin{tabular}{|c|c|c|c|c|}
\hline Compound & R1 & R2 & M.P & Yield (\%) \\
\hline $\mathbf{3 a}$ & CF3 & C2H5 & 117 & 46.2 \\
\hline $\mathbf{3 b}$ & CF3 & 4CH3- phenyl & 156 & 52.3 \\
\hline $\mathbf{3 c}$ & CF3 & 4CF3- phenyl & 124 & 49.5 \\
\hline $\mathbf{3 d}$ & 4CH3- phenyl & C2H5 & 172 & 58.3 \\
\hline $\mathbf{3 e}$ & 4CF3- phenyl & C2H5 & 228 & 55.7 \\
\hline $\mathbf{3 f}$ & 2,5- Dichloro phenyl & C2H5 & 152 & 65.3 \\
\hline $\mathbf{3 g}$ & CF3 & C2H5 & 196 & 63.4 \\
\hline $\mathbf{3 h}$ & CF3 & 4CH3- phenyl & 188 & 58.4 \\
\hline $\mathbf{3 i}$ & CF3 & 4CF3- phenyl & 207 & 48.9 \\
\hline $\mathbf{3 j}$ & 4CH3- phenyl & C2H5 & 218 & 40.2 \\
\hline $\mathbf{3 k}$ & 4CF3- phenyl & C2H5 & 202 & 61.3 \\
\hline $\mathbf{3 i}$ & 2,5- Dichloro phenyl & C2H5 & 221 & 39.4 \\
\hline
\end{tabular}

Table 1: List of Synthesized compound.

\begin{tabular}{|c|c|c|c|c|}
\hline Compound & S. aureus & S. pyogenus & E. coli & P. aeruginosa \\
\hline 3a & 100 & 200 & 200 & 200 \\
\hline 3b & 62.5 & 125 & 200 & 250 \\
\hline 3c & 100 & 100 & 200 & 200 \\
\hline 3d & 250 & 100 & 62.5 & 125 \\
\hline 3e & 125 & 200 & 125 & 100 \\
\hline 3f & 100 & 100 & 100 & 200 \\
\hline $3 \mathbf{3 g}$ & 250 & 200 & 125 & 250 \\
\hline $3 \mathbf{3 h}$ & 250 & 200 & 250 & 500 \\
\hline $3 \mathbf{3 i}$ & 200 & 200 & 250 & 250 \\
\hline $3 \mathbf{3 j}$ & 100 & 250 & 100 & 250 \\
\hline 3k & 250 & 200 & 250 & 100 \\
\hline 3I & 100 & 200 & 250 & 100 \\
\hline Ampicillin & 250 & 100 & 100 & 100 \\
\hline Chloramphenicol & 50 & 50 & 50 & 50 \\
\hline
\end{tabular}

Table 2: Antibacterial activity of sulfonamide and amide derivatives (In MIC).

\begin{tabular}{|c|c|c|c|}
\hline Compound & C. albicans & A. niger & A. clavatus \\
\hline $\mathbf{3 a}$ & 250 & $>1000$ & $>1000$ \\
\hline $\mathbf{3 b}$ & 1000 & 1000 & 1000 \\
\hline $\mathbf{3 c}$ & 500 & 250 & 500 \\
\hline $\mathbf{3 d}$ & 1000 & $>1000$ & $>1000$ \\
\hline $\mathbf{3 e}$ & 500 & $>1000$ & $>1000$ \\
\hline $\mathbf{3 f}$ & 1000 & 500 & 1000 \\
\hline $\mathbf{3 g}$ & 1000 & $>1000$ & $>1000$ \\
\hline $\mathbf{3 h}$ & 500 & 500 & 250 \\
\hline $\mathbf{3 i}$ & 500 & 500 & 250 \\
\hline $\mathbf{3 j}$ & 1000 & 1000 & 1000 \\
\hline $\mathbf{3 k}$ & $>1000$ & 500 & 500 \\
\hline $\mathbf{3 l}$ & 500 & $>1000$ & $>1000$ \\
\hline Nystatin & 100 & 100 & 100 \\
\hline Greseofulvin & 500 & 100 & 100 \\
\hline & \multicolumn{2}{|c|}{ Table 3: Antifungal activity (in MIC). } \\
\hline
\end{tabular}

\section{Conclusion}

All the newly synthesized compounds were screened for antibacterial, antifungal and antimalarial activity. The data in the Tables 2 and 3 indicate that among the synthesized compound $3 \mathrm{a}, 3 \mathrm{~b}$ and $3 \mathrm{c}$ possesses good antimicrobial activity. However, the activities of the tested compounds are much less than those of standard agents used. These compounds also show potent antimalarial activity. From the results of various biological activities it is clear that these compounds would be of better use in drug development to combat bacterial infections and as antimalarial agents in the future.

\begin{tabular}{|c|c|}
\hline Compound & $\begin{array}{c}\text { Mean } \mathbf{I C}_{\mathbf{5 0}} \\
\text { (Micrograme/ml) }^{\text {(Micro }}\end{array}$ \\
\hline $\mathbf{3 a}$ & 0.77 \\
\hline $\mathbf{3 b}$ & 0.97 \\
\hline $\mathbf{3 c}$ & 0.79 \\
\hline $\mathbf{3 d}$ & 0.80 \\
\hline $\mathbf{3 e}$ & 0.89 \\
\hline $\mathbf{3 f}$ & 0.98 \\
\hline $\mathbf{3 g}$ & 1.01 \\
\hline $\mathbf{3 h}$ & 1.12 \\
\hline $\mathbf{3 i}$ & 0.98 \\
\hline $\mathbf{3 j}$ & 1.12 \\
\hline $\mathbf{3 k}$ & 0.79 \\
\hline $\mathbf{3 i}$ & 0.96 \\
\hline Quinine & 0.268 \\
\hline
\end{tabular}

Table 4: Antimalarial activity.

\section{Acknowledgements}

The authors would like to thank to Dr. Dhanji P. Rajani and Mr. Kalpesh of Microcare Laboratory, and Tuberclosis Research Centre, Surat for conducting the antibacterial, antifungal and antimalarial activity.

\section{References}

1. Genc $Y$, Ozkanca R, Bekdemir Y (2008) Antimicrobial activity of some sulfonamide derivatives on clinical isolates of Staphylococus aureus. Ann Clin Microbiol Antimicrob 7: 17-22

2. Ozbek N, KatircioÄŸlu H, Karacan N, Baykal T (2007) Synthesis, characterization and antimicrobial activity of new aliphatic sulfonamide. Bioorg Med Chem 15: 5105-5109.

3. Mun J, Jabbar AA, Devi NS, Yin S, Wang Y, et al. (2012) Design and in-vitro activities of $\mathrm{N}$-alkyIN-[(8-R-2,2-dimethyl-2H-chromen-6-yl)methyl] hetero aryl sulfonamides, novel, small-molecule hypoxia inducible factor-1 pathway inhibitors and anticancer agents. J. Med. Chem. 55: 6738-6750.

4. El-Sayed NS, El-Bendary ER, El-Ashry SM, El-Kerdawy MM (2011) Synthesis and antitumor activity of new sulfonamide derivatives of thiadiazolo[3,2-a] pyrimidines. Eur J Med Chem 46: 3714-3720.

5. Borne RF, Peden RL, Waters IW, Weiner M, Jordan R, et al. (1974) Antiinflammatory activity of para-substituted $\mathrm{N}$-benzenesulfonyl derivatives of anthranilic acid. J Pharm Sci 63: 615-617.

6. De Clercq E (2001) New developments in anti-HIV chemotherapy. Curr Med Chem 8: 1543-1572.

7. Bhusari KP, Khedekar PB, Umathe SN, Bahekar RH, Rao ARR (2000) Synthesis of 8-bromo-9-substituted 1,3-benzothiazolo-[5,1-b]-1,3,4-triazoles and their anthelmintic activity. Indian J. Heterocyclic Chem 9: 275-278.

8. Bhusare SR, Pawar RP, Vibhute YB (2001) Synthesis and antibacterial activity of some new 2-(substituted phenyl sulfonamido)-6-substituted benzothiazoles. Indian J Heterocyclic Chem 11: 79-80.

9. Ahmed B, Khan SA, Alam T (2003) Synthesis and antihepatotoxic activity of some heterocyclic compounds containing the 1,4-dioxane ring system. Pharmazie 58: 173-176.

10. Shekar BC, Roy K, De AU (2001) Synthesis of some new p-toluene sulfonamido glutaramides. Indian J. Heterocyclic Chem. 10: 237-238.

11. Tambem T, Guillem $\vee$ (1998) Adrenomedullin and related peptides. Folia pharmacol. Jpn. 112: 138-142.

12. Volberding PA, Mitsuyasu RT, Golando JP, Spiegel RJ (1987) Treatment of Kaposi's sarcoma with interferon alfa-2b (Intron A). Cancer 59: 620-625.

13. Antoine M (1991) Benzo[1,8]naphthyridine derivatives, their preparation and compositions containing them. Michel Laboratorie Roger Bellon, US-5053509.

14. Nakazato A, Chaki S, Okubo T, Shin-Ichi O, Ishii T (2002) Remedial agent for anxiety neurosis or depression and piperazine derivative. Taisho Pharmaceutical Co, WO-0200259.

15. Shue HJ (1998) Piperazino derivatives as neurokinin antagonists. Schering Corporation WO-9808826. 
16. Middlemiss D, Judkins BD, Eldred CD, Porter B, Kelly HA (1993) 1-piperazineacetic derivatives as fibrinogen receptor antagonists. Research report, GlaxoSmithkline WO-9322303.

17. Meyer WE, Tomcufcik AS, Chan PS, Haug M (1989) 5-(1-piperazinyl)-1H-1,2,4triazol-3-amines as antihypertensive agents. J Med Chem 32: 593-597.

18. Carceller E, Almansa C, Merlos M, Giral M, Bartrola J, et al. (1992) (Pyridylcyanomethyl)piperazines as orally active PAF antagonists. J Med Chem 35: 4118-4134.

19. Carceller E, Merlos M, Giral M, Almansa C, Bartrola J, et al. (1993) Synthesis and structure-activity relationships of 1-acyl-4-((2-methyl-3-pyridyl)cyanomethyl) piperazines as PAF antagonists. J Med Chem 36: 2984-2997.

20. Herrin TR, Pauvlik JM, Schuber EV, Geiszler AO (1975) Antimalarials. Synthesis and antimalarial activity of 1-(4-methoxycinnamoyl)-4-(5-phenyl-4oxo-2-oxazolin-2-yl)piperazine and derivatives. J Med Chem 18: 1216-1223.

21. Kimura M, Masuda T, Yamada K, Kubota N, Kawakatsu N, et al. (2002) Novel diphenylalkyl piperazine derivatives with dual calcium antagonistic and antioxidative activities. Bioorg Med Chem Lett 12: 1947-1950.

22. Upadhayaya RS, Sinha N, Jain S, Kishore N, Chandra R, et al. (2004) Optically active antifungal azoles: synthesis and antifungal activity of $(2 R, 3 S)-2-(2,4-$ difluorophenyl)-3-(5-[2-[4-aryl-piperazin-1-yl]-ethyl]-tetrazol-2-yl/1-yl)-1-[1,2,4]triazol-1-yl-butan-2-ol. Bioorg Med Chem 12: 2225-2238.

23. Kumar Parai M, Panda G, Srivastava K, Kumar Puri S (2008) Design, synthesis and antimalarial activity of benzene and isoquinoline sulfonamide derivatives. Bioorg. Med. Chem. Lett 18: 776-781.

24. Asundaria S, Shahrukh T, Patel L, Keshav C (2010) Synthesis and characterization of some sulfonamide based bis-sydnones and their in vitro antimicrobial activity. Indian J. Chem Sect. B 49: 960-964

25. Suchetan PA, Mohan RN, Vijith K, Palakshamurthy BS, Sreenivasa S (2013) 6-Chloro-N-(pyridin-4-ylmethyl)pyridine-3-sulfonamide. Acta Crystl. 69: 01765.

26. El-Din NS (2000) Synthesis of some sulfonamide derivatives with potential antibacterial activity. Chem. Heterocycl. Compd 36: 449-454.

27. Ananda Kumar CS, Nanjunda Swamy S, Thimmegowda NR, Benaka Prasad SB, Yip GW (2007) Synthesis and evaluation of 1-benzhydryl-sulfonylpiperazine derivatives as inhibitors of MDA-MB-231 human breast cancer cell proliferation. Med. Chem. Res 16: 179-187.

28. Saingar S, Kumar R, Joshi YC (2011) Synthesis and biological activity of novel $1 \mathrm{H}-1,4-d i a z e p i n e s$ containing benzene sulfonyl piperazine moiety. Med. Chem. Res. 20: 975-980.

29. Mallesha L, Mohana KN (2011) Synthesis, antimicrobial and antioxidant activities of 1-(1,4-benzodioxane-2-carbonyl)piperazine derivatives. Eur. J. Chem. 2: 193-199.

30. Al-Soud YA, Al-Sa'doni HH, Amajaour HAS, Salih KSM, Mubarak MS (2008) Synthesis, characterization and anti-HIV and antitumor activities of new coumarin derivatives. Chem. Sci. 63: 83-89.

31. Martyn DC, Cortese JF, Tyndall E, Dick J, Mazitschek R, et al. (2010) Antiplasmodial activity of piperazine sulfonamides. Bioorg. Med. Chem. Lett. 20: $218-221$

32. Moghazy Aly SME, Georgey HH, Mohammed MA, Gawad NAM, Amin NH (2007) Synthesis and antineoplastic activity of certain triazene and Triazeno -Acridine combilexin derivatives. Bull. Pharm. Sci. 30: 89-110.

33. Al-Soud YA, Al-Sa'doni HH, Bahjat S, Jaber IH, Beni-Khalid M, et al. (2008) Synthesis and in vitro antiproliferative activity of new benzothiazole derivatives. ARKIVOC 15: 225-238

34. Hayat F, Yoo E, Rhim H, Choo HYP (2013) Synthesis and Inhibition Effects on 5-HT6 Receptor of Benzothiazole Derivatives. Bull. Korean Chem. Soc. 34: 495-499.

35. Yoon J, Yoo EA, Kim JY, Pae AN, Rhim H, et al. (2008) Preparation of piperazine derivatives as 5-HT7 receptor antagonists. Bioorg Med Chem 16: 5405-5412.

36. Borrmann T, Hinz S, Bertarelli DC, Li W, Florin NC, et al. (2009) 1-alkyl-8(piperazine-1-sulfonyl)phenylxanthines: development and characterization of adenosine $\mathrm{A} 2 \mathrm{~B}$ receptor antagonists and a new radioligand with subnanomolar affinity and subtype specificity. J Med Chem 52: 3994-4006.

37. Wang Y, Busch-Petersen J, Wang F, Kiesow TJ, Graybill TL, et al. (2009)
Camphor sulfonamide derivatives as novel, potent and selective CXCR3 antagonists. Bioorg Med Chem Lett 19: 114-118.

38. Xiang J, Wan ZK, Li HQ, Ipek M, Binnun E, et al. (2008) Piperazine sulfonamides as potent, selective, and orally available 11 beta-hydroxysteroid dehydrogenase type 1 inhibitors with efficacy in the rat cortisone-induced hyperinsulinemia model. J Med Chem 51: 4068-4071.

39. Oh S, Moon HI, Son IH, Jung JC, Avery MA (2007) Synthesis of sulfonamides and evaluation of their histone deacetylase (HDAC) activity. Molecules 12: $1125-1135$.

40. Cumming J, Babu S, Huang Y, Carrol C, Chen X, et al. (2010) Piperazine sulfonamide BACE1 inhibitors: design, synthesis, and in vivo characterization. Bioorg. Med. Chem. Lett. 20: 2837-2842.

41. Timothy PH, Megan B, Georgette C, Christine C, Irina C, et al (2010) Identification of GNE-477, a potent and efficacious dual PI3K/mTOR inhibitor Bioorg. Med. Chem. Lett. 20: 2408-2411.

42. Kandile NG, Mohamed MI, Zaky H, Mohamed HM (2009) Novel pyridazine derivatives: synthesis and antimicrobial activity evaluation. European Journal of Medicinal Chemistry. 44:1989-1996.

43. Asif M, Singh A, Ratnakar L (2011) Antimicrobial agents: brief study of pyridazine derivatives against some phathogenic microorganisms. Journal of Pharmacy Research. 4: 664-667.

44. Benmoussa A, El harti J, Ansar M, Bouchrik M, Zahidi A et al. (2012) Synthesis and antimicrobial properties of some pyridazin-3-thiones derivatives. International Journal of Pharm Tech Research 4: 1591-1594.

45. Mangalagiu II (2011) Recent achievements in the chemistry of 1,2diazines Current Organic Chemistry 15: 730-752.

46. Mantu D, Luca MC, Moldoveanu C, Zbancioc G, Mangalagiu II (2010) Synthesis and antituberculosis activity of some new pyridazine derivatives. Part II. European Journal of Medicinal Chemistry 45: 5164-5168.

47. Butnariu RM, Mangalagiu II (2009) New pyridazine derivatives: synthesis, chemistry and biological activity. Bioorg Med Chem 17: 2823-2829.

48. Drochioiu G, Sunel V, Oniscu C, Basu C, Murariu M (2001) The break down of plant bio structure followed by aminoacids determination. Romanian Biotechnological Letters 6:155-165.

49. Butnariu RM, Caprosu MD, Bejanetal V (2007) Pyridazine and phthalazine derivatives with potential antimicrobial activity. Journal of Heterocyclic Chemistry 4: 1149-1152.

50. Dima S, Caprosu M, Ungureanu M, Grosu G, Petrovanu M (1999) New derivatives of 1-methyl-phthalazine with antimicrobial and fungistatic action. Annales Pharmaceutiques Francaises 57: 415-416.

51. Han X, Hong YH, You Quan Z, Xiao Mao Z, Bin L, et al. (2010) Synthesis and herbicidal activities of novel 4-(3-trifluoromethylphenyl)-2H-pyridazin3-one derivatives. Science China Chemistry 53: 157-166.

52. Refaat HM, Khalil OM, Kadry HH (2007) Synthesis and anti inflammatory activity of certain piperazinyl thienyl pyridazine derivatives. Archives of Pharmacal Research 30: 803-811.

53. Liljebris C, Martinsson J, Tedenborg L, Williamsa M, Barker E, et al. (2002) Synthesis and biological activity of a novel class of pyridazine analogues as non-competitive reversible inhibitors of protein tyrosine phosphatase1B (PTP1B). Bioorganic and Medicinal Chemistry 10: 3197-3212.

54. Tucaliuc RB, Risca IM, Drochioiu G, Mangalagiu I (2008) Biological effect of some new pyridazine derivatives on wheat in germination experiments. Romanian Biotechnological Letters 13: 3837-3842.

55. Stanovnik B, Tisler M (1967) Synthesis of pyridazine derivatives. 13. Formation of some substituted imidazo (1.2-b) pyridazines. Tetrahedron 23: 2739-2746.

56. Almirante L, Polo L, Mugnaini A, Provinciali E, Rugarli P, et al. (1966) Derivatives of imidazole. II. Synthesis and reactions of imidazo[1,2-alpha]pyrimidines and other bi- and tricyclic imidazo derivatives with analgesic, antiinflammatory, antipyretic, and anticonvulsant activity. J Med Chem 9: 29-33.

57. Japanese Patent 22,264 (1965); Chem. Abstr., 64, 3566 (1966)

58. Japanese Patent 22,265 (1965); Chem. Abstr., 64, 3566 (1966).

59. Japanese Patent 22,263 (1965); Chem. Abstr., 64, 3566 (1966). 
Citation: Bhatt A, Kant R, Singh RK (2016) Synthesis of Some Bioactive Sulfonamide and Amide Derivatives of Piperazine Incorporating Imidazo[1,2-B] Pyridazine Moiety. Med chem (Los Angeles) 6: 257-263. doi:10.4172/2161-0444.1000355

60. Henry D. Isenberg. Clinical microbiology procedure handbook. (2nd edn.), Volume II, Chapter 5.

61. Desai NC, Shihora PN, Moradia DL (2007) Synthesis and characterization of new quinazolines as potential antimicrobial agents. Indian journal of chemistry 46B: 550-553.
62. Shadomy S (1991) In Manual of Clinical Microbiology. ASM Press: Washington, DC, p: 1173

63. Rattan A (2000) Antimicrobials in Laboratory Medicine. BI Churchill Livingstone India, p: 85. 